\title{
Genetic variation in four maturity genes affects photoperiod insensitivity and PHYA-regulated post-flowering responses of soybean
}

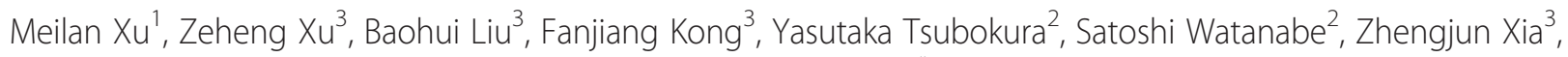
Kyuya Harada ${ }^{2}$, Akira Kanazawa', Testuya Yamada ${ }^{1}$ and Jun Abe ${ }^{1 *}$

\begin{abstract}
Background: Absence of or low sensitivity to photoperiod is necessary for short-day crops, such as rice and soybean, to adapt to high latitudes. Photoperiod insensitivity in soybeans is controlled by two genetic systems and involves three important maturity genes: E1, a repressor for two soybean orthologs of Arabidopsis FLOWERING LOCUS T (GmFT2a and GMFT5a), and E3 and E4, which are phytochrome A genes. To elucidate the diverse mechanisms underlying photoperiod insensitivity in soybean, we assessed the genotypes of four maturity genes (E1 through E4) in early-flowering photoperiod-insensitive cultivars and their association with post-flowering responses.

Results: We found two novel dysfunctional alleles in accessions originally considered to have a dominant E3 allele according to known DNA markers. The E3 locus, together with E1 and E4, contained multiple dysfunctional alleles. We identified 15 multi-locus genotypes, which we subdivided into 6 genotypic groups by classifying their alleles by function. Of these, the e1-as/e3/E4 genotypic group required an additional novel gene (different from E1,E3, and E4) to condition photoperiod insensitivity. Despite their common pre-flowering photoperiod insensitivity, accessions with different multi-locus genotypes responded differently to the post-flowering photoperiod. Cultivars carrying E3 or E4 were sensitive to photoperiod for post-flowering characteristics, such as reproductive period and stem growth after flowering. The phytochrome A-regulated expression of the determinate growth habit gene Dt1, an ortholog of Arabidopsis TERMINAL FLOWER1, was involved in the persistence of the vegetative activity at the stem apical meristem of flower-induced plants under long-day conditions.
\end{abstract}

Conclusions: Diverse genetic mechanisms underlie photoperiod insensitivity in soybean. At least three multi-locus genotypes consisting of various allelic combinations at E1,E3, and E4 conferred pre-flowering photoperiod insensitivity to soybean cultivars but led to different responses to photoperiod during post-flowering vegetative and reproductive development. The phyA genes E3 and E4 are major controllers underlying not only pre-flowering but also post-flowering photoperiod responses. The current findings improve our understanding of genetic diversity in pre-flowering photoperiod insensitivity and mechanisms of post-flowering photoperiod responses in soybean.

Keywords: Photoperiod, Soybean, Flowering, Determinate habit, Post-flowering, Genetic variation

\footnotetext{
* Correspondence: jabe@res.agr.hokudai.ac.jp

${ }^{1}$ Research Faculty of Agriculture, Hokkaido University, Sapporo 060-8589, Japan

Full list of author information is available at the end of the article
} 


\section{Background}

Photoperiod sensitivity is an important trait that enables crops to adapt to diverse latitudinal environments. In particular, absence of or low sensitivity to photoperiod is necessary for short-day (SD) crops, such as rice and soybean, to adapt to high latitudes. In soybean (Glycine max (L.) Merr.), nine major genes, $E 1$ through $E 8$ and $J$, have so far been reported to control time to flowering and maturity (reviewed in [1]). Photoperiod insensitivity is controlled by at least two genetic systems, in which dysfunctional alleles at three maturity loci-E1, E3, and E4are involved. One control system is ascribed to the double-recessive genotype for $E 3$ and $E 4$ [2-6], which encode the phytochrome A (PHYA) proteins GmPHYA3 and GmPHYA2, respectively $[7,8]$. Together with GmPHYA1, which is encoded by a homoeologous copy of E4, these two PHYA proteins redundantly or complementarily function in floral induction and de-etiolation responses under various light conditions [7,8]. E3 and E4 direct different flowering responses to long-day (LD) conditions with different red-to-far-red (R:FR) quantum ratios [2-5,9]. E3 controls the response to light with a high or low R:FR ratio; plants homozygous for the recessive $e 3$ allele can initiate flowering under the LD conditions generated by fluorescent lamps with a high R:FR ratio [2,9]. E4 is involved in the response to light with a low R:FR ratio; plants homozygous for the $e 3$ allele need a recessive $e 4$ allele to flower under LD generated by incandescent lamps with a low R:FR ratio $[3,4,9]$. The $e 4$ allele itself cannot confer the insensitivity to LD conditions induced by both fluorescent and incandescent lamps under the E3 genetic background $[4,5]$. The PHYA protein is not only an effective FR sensor but also acts as an R-light photoreceptor under $\mathrm{R}$ light with high-photon irradiance $[10,11]$, which is involved, either directly or via interactions with other photoreceptors, in various developmental processes (reviewed in [12]). The E3 and E4 genes therefore may participate in a non-additive manner in different aspects of PHYA functions, which are controlled by a single phyA gene in Arabidopsis. Another phyA gene in soybean, GmphyA1, has been suggested to function redundantly with $E 4$ in the de-etiolation response of hypocotyls and floral induction under FR light [7]. Owing to the lack of genetic variants causing phenotypic differences, the function of GmphyA1 has not yet been determined.

Among the major genes and QTLs that have been reported so far, the $E 1$ gene has the most prominent effect on flowering time in soybean (reviewed in [1]). Cober et al. [9] used an early-maturing cultivar Harosoy and its nearisogenic lines (NILs) for E1, E3 and E4 loci to reveal their photoperiod responses to LDs with different R:FR ratios. They found that a NIL with E1/e3/e4 was insensitive to R-enriched LD conditions but still retained sensitivity to FR-enriched LD conditions with the low R:FR ratio of
0.9 , although the NIL with $e 1$-as (originally designed as $e 1$, but renamed after [13])/e3/e4 lost the sensitivity across the wide range of R:FR ratios [9]. This result indicates that E1 has a marked inhibitory effect on flowering, particularly under LD conditions with the low R:FR ratio.

Positional cloning revealed that $E 1$ encodes a protein that contains a putative bipartite nuclear localization signal and a region related to the B3 domain, a highly conserved domain found in transcription factors in plants [13]. The abundance of $E 1$ transcripts is under the photoperiodic control regulated by the $E 3$ and $E 4$ genes and is negatively correlated with that of two soybean orthologs of Arabidopsis FLOWERING LOCUS T, GmFT2a and GmFT5a [14]. E1 expression was suppressed under SD conditions regardless of genotype and induced under LD conditions in plants containing either $E 3$ or E4 but not in those with the double-recessive e3e3e4e4 genotype [13]. Furthermore, $E 1$ over-expression in transgenic T2 soybean plants suppressed the expression of two GmFT genes, thereby markedly delaying flowering, suggesting that E1 is a direct repressor of GmFTs [13]. Taken together, the weakened function of PHYA caused by the double-recessive genotype at the $E 3$ and $E 4$ loci may ablate or weaken sensitivity to photoperiod, at least partly, through the downregulation of $E 1$.

The other system controlling photoperiod insensitivity in soybean acts through involvement of dysfunctional alleles at the $E 1$ locus itself. Photoperiod insensitivity in the Japanese landrace 'Sakamotowase' has been suggested to be controlled by a different genetic mechanism from PHYA dysfunction, because this landrace has a dominant functional allele at the E4 locus [6]. Quantitative trait locus (QTL) mapping using testcrosses with a photoperiod-sensitive Harosoy NIL whose genotype was e1-as/e3/E4 indicated that the photoperiod insensitivity in Sakamotowase was controlled mainly by an allele at or a gene tightly linked to the $E 1$ locus, although a minor QTL was detected in linkage group L (Glyma19) [15]. Analysis of the E1 sequence of Sakamotowase revealed that it contained a dysfunctional allele, $e 1-f s$, that produced a loss-of-function truncated protein due to a premature stop codon, which arose as a consequence of a single-base deletion [13]. In contrast, the recessive allele e1-as possessed a nonsynonymous substitution in the putative nuclear localization signal, leading to reduced localization specificity of the E1 protein in nucleus and thereby reducing the ability of E1 to suppress expression of the GmFT genes [13]. These findings strongly suggest that the molecular basis of the photoperiod insensitivity of Sakamotowase is due, at least in part, to a complete lack of E1 function caused by a dysfunctional allele at the $E 1$ locus itself. The dysfunctional allele $e 1-f s$ therefore may provide, singly or together with other unknown genes, another mechanism in the control of 
photoperiod insensitivity under the presence of E4 in soybean.

Photoperiod responses of soybean involve not only preflowering growth, such as time to flowering, but also postflowering vegetative and reproductive growth, such as duration of pod filling, development of the terminal inflorescence, and leaf senescence; LD conditions increase reproductive periods and delay leaf senescence and seed maturation of photoperiod-sensitive cultivars [16-18]. Han et al. [16] revealed that the post-flowering growth of a photoperiod-sensitive cultivar was influenced by exposure to $\mathrm{R}$ light and reversed by exposure to FR light during the dark period, suggesting that phytochromes control the photoperiod responses of post-flowering growth. However, understanding of the genetic and physiologic bases of post-flowering photoperiod sensitivity is still far from comprehensive.

Soybean maturity genes control not only the time of flowering but also the time to maturation [19-21]. Several QTLs in various linkage groups have been identified to control features of the reproductive period, such as time of maturity and duration of pod filling [22-26]. However, only a few QTLs for post-flowering photoperiod responses have been identified. Cheng et al. [25] found two QTLs that controlled the duration of the post-flowering period under LD conditions. These two QTLs, which appear to correspond to the $E 3$ and $E 8$ [27] genes, also were involved in the control of time to flowering [25].

To survey the diverse mechanisms underlying photoperiod insensitivity in soybean and to search for novel genetic factors involved in this process, we first used allelespecific DNA markers to catalog the genotypes of four maturity genes (E1 through $E 4$ ) in photoperiod-insensitive cultivars and breeding lines introduced from various geographic regions. We also tested the association of these various genotypes with pre- and post-flowering photoperiod responses. Here we report that soybean has gained the trait of photoperiod insensitivity independently and repeatedly through diverse mechanisms. In addition, $E 3$ and $E 4$, together with $E 1$, play important roles in not only floral initiation but also post-flowering photoperiod responses, such as maturation and stem termination, in soybean.

\section{Results}

\section{Effects of photoperiod insensitivity on flowering}

As in our previous studies [6,15], we evaluated photoperiod sensitivity according to the difference in the time to flowering (stage R1 [28]) between artificially induced LD and natural daylength (ND) conditions. Harosoy is a photoperiod-sensitive, early-maturing indeterminate cultivar: it possesses a maturity genotype of e1-as/e2/E3/E4, and a dominant Dt1 gene [5]. During the 2-year study period, this cultivar flowered at an average of 57 days after sowing (DAS) under ND conditions of Sapporo, Japan $\left(43^{\circ} 06^{\prime} \mathrm{N}, 141^{\circ} 35^{\prime} \mathrm{E}\right)$ but did not produce any flower buds during LD until the end of the artificially induced condition (62 to 65 DAS). Similarly, Harosoy NILs for e3 (H-e3) or e4 (H-e4) did not form any flower buds until the end of the artificially induced LD condition, and they flowered slightly earlier during ND conditions than did Harosoy; the average flowering time was 46 DAS for $\mathrm{H}-e 3$ and 53 DAS for $\mathrm{H}-e 4$. In contrast, the Harosoy NIL for both $e 3$ and e4 (H-e3/e4) flowered at 40 DAS under both ND and LD, and this line produced pods of $2 \mathrm{~cm}$ or longer (stages R4 to R5 [28]) by the end of the artificially induced LD condition. All 53 photoperiodinsensitive accessions used in the current study flowered under LD conditions within 5 days after the date that they flowered under ND conditions.

\section{Classification of genotypes by use of allele-specific DNA markers}

The molecular bases of four maturity genes (E1 through $E 4)$ have been determined $[7,8,13,29]$. To determine the allelic constitutions at the four loci, we genotyped the 4 genes in each of the 53 photoperiod-insensitive accessions by using previously reported allele-specific DNA markers [7,13,29-31].

Four alleles have been identified at the E1 locus [13]. These alleles include two null alleles: one lacking a 130-kb region harboring the entire gene $(e 1-n l)$ and the other having a single-base deletion that leads to a frameshift mutation that generates a premature stop codon $(e 1-f s)$. The conventional recessive allele $e 1$ (here designated as e1-as according to [13]) differs from the dominant allele (E1) by a single amino acid substitution in the putative nuclear localization signal; this substitution abolishes nuclear localization of the protein. Eight (seven Japanese and one Chinese) of the 53 accessions had the E1 allele, 33 had $e 1-a s$, and 11 had $e 1-n l$; only the Japanese landrace 'Sakamotowase' had the $e 1-f s$ allele (Additional file 1).

Two alleles of the E2 gene, a soybean ortholog of Arabidopsis GIGANTEA (GI), have been identified: a functional dominant allele $(E 2)$ and a recessive null allele $(e 2)$ [29]. All of the 53 accessions we tested had the $e 2$ allele (Additional file 1).

Two and six alleles have previously been identified at the $E 3$ and E4 loci, including one (e3) and five (e4-SORE-1, e4-kam, e4-oto, e4-tsu, and e4-kes) null alleles of the E3 and $E 4$ loci, respectively [7,8,31]. Of the 53 accessions, 20 had the $E 3$ allele, and the remaining 33 accessions had the $e 3$ allele. Fifteen accessions had $E 4$, and the remaining 38 accessions had one of four dysfunctional alleles: e4-SORE1 (20), e4-kam (2), e4-oto (1), and e4-kes (15) (Additional file 1). Only 22 accessions were double-recessive homozygotes for the $E 3$ and $E 4$ loci, whereas 27 accessions had either of the two dominant alleles, and 4 had both. 


\section{Sequencing of the $E 1, E 3$, and $E 4$ genes}

Genotyping of the four maturity genes by using allelespecific markers indicated that, despite their photoperiod insensitivity, 31 accessions had dominant alleles at either or both of the E3 and E4 loci. This result suggests that a novel allele or gene may be involved in the control of this trait. We then sequenced the accessions having at least one dominant E1,E3, or E4 allele to examine whether these alleles were truly functional. No sequence variation was detected in the coding region of $E 1$ in the eight accessions of the E1/e3/e4 genotype; all showed the same sequence as the published dominant E1 allele [13]. Sequence analysis of the E4 gene similarly revealed no deviations from the published sequence [7] in the 15 accessions tested. However, we found two novel dysfunctional alleles for the E3 gene among the 18 accessions initially scored as having the $e 1 / E 3 / E 4$ or $e 1 / E 3 / e 4$ genotype (Figure 1A and $1 \mathrm{~B})$. One of these novel alleles was a nonsense mutation, in which a single-nucleotide substitution from $C$ to $\mathrm{T}$ at position 3139 from the adenine of the first codon in exon 3 created a stop codon in place of a codon encoding glutamine. The other novel allele had a premature stop codon in exon 1 that was generated by frameshifting due to the insertion of $\mathrm{T}$ at position 1275 in exon 1. We designated these dysfunctional alleles $e 3-$ $n s$ and $e 3-f s$, respectively, and renamed the conventional $e 3$ allele as e3-tr (truncated), because it lacks the 3' region of the gene, including exon 4 [8].

We then developed allele-specific cleaved amplified polymorphic sequence (CAPS) or derived CAPS (dCAPS) markers to reliably identify these dysfunctional alleles (Figure 1C). The $e 3-f s$ allele can be distinguished from the others after treatment with AleI, which cleaves the 758- or 759-bp amplified product flanking the mutation site into fragments of 552 and $206 \mathrm{bp}$ for the E3, e3-ns, and $e 3-t r$ alleles but not $e 3-f s$ (Figure 1C). The $e 3-n s$ allele can be distinguished from the others after treatment with $M f e I$, which uniquely digests the 163-bp amplified e3-ns product into fragments of 140 and $23 \mathrm{bp}$. Most of the accessions, which were originally thought to contain the E3 allele, had dysfunctional alleles and thus produced truncated GmPHYA3 proteins.

We classified the 53 photoperiod-insensitive accessions into 15 multi-locus genotypes, and then grouped the alleles by function to subcategorize the accessions into 6 genotypic groups (e1/e3/e4, e1/E3/e4, e1/e3/E4, e1-as/ $e 3 / e 4, e 1-a s / e 3 / E 4$, and E1/e3/e4), where e1, e3, and e4 refer to loss-of-function alleles at the respective locus
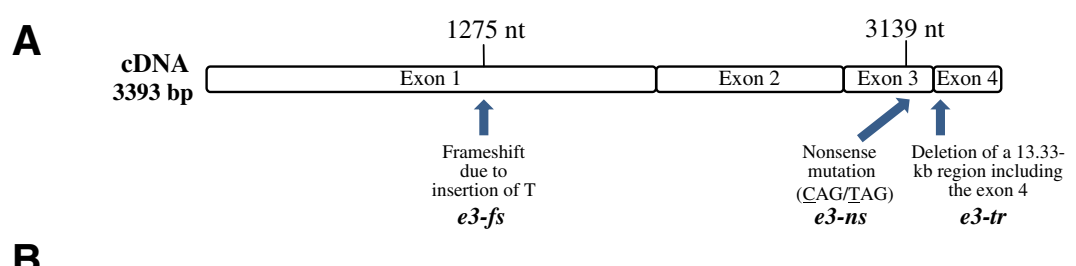

B
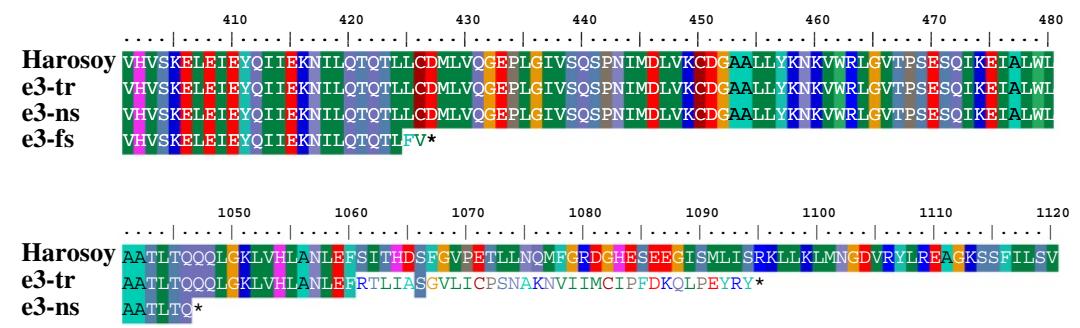

C


Figure 1 Novel null alleles detected at the E3 locus in photoperiod-insensitive soybean accessions. A) Positions and types of mutations. B) Changes in predicted amino acid sequences; asterisks indicate stop codons. C) DNA markers distinguishing each of the recessive alleles from the others. Cultivars Darta and Nawiko have e3-fs and e3-ns, respectively. Harosoy has a dominant E3 allele. Marker diagnostic for e3-fs shown in left panel. Marker diagnostic for e3-ns shown in right panel. nd, not digested. 
(Table 1). Of these, a combination of dysfunctional alleles at both the E3 and E4 loci was predominant: 36 of the 53 accessions shared this genotype. Of the 17 accessions with either the E3 or E4 allele, 10 had $e 1-f s$ or $e 1-n l$ at the E1 locus. The remaining seven accessions had the allelic combination of $e 1$-as/e3/E4, the same genotype as that of $\mathrm{H}-e 3$, which is sensitive to the long days generated by an FR-enriched light source $[9,15]$, such as the incandescent lamps we used in the current study.

Collectively, all of the 53 photoperiod-insensitive accessions analyzed had a recessive allele at either the E3 or E4 locus. When one of these loci had a dominant allele, the E1 locus always had a loss-of-function e1-fs or $e 1-n l$ allele or the hypomorphic $e 1$-as allele.

\section{Post-flowering photoperiod sensitivity associated with E3 and E4}

The 53 accessions we tested were considered to be photoperiod insensitive in terms of time to flowering, because the difference in flowering dates between ND and LD conditions was small (that is, 5 days or less). However, these accessions differed markedly in their post-flowering vegetative and reproductive growth characteristics, such as reproductive period and stem growth after flowering (Figures 2 and 3). Two-way analysis of variance, in which a combined mean square for interactions by years was used as an error mean square, revealed highly significant $(P<0.001)$ differences for both traits among accessions, daylength conditions, and their interaction.

\begin{tabular}{|c|c|c|c|c|c|}
\hline \multirow{2}{*}{$\begin{array}{l}\text { Multi-locus } \\
\text { genotype }\end{array}$} & \multicolumn{4}{|c|}{ Allelic combinations at four maturity loci } & \multirow{2}{*}{$\begin{array}{c}\text { No. of } \\
\text { accessions }\end{array}$} \\
\hline & El & E2 & E3 & E4 & \\
\hline el/e3/e4 & $e l-n l$ & $e 2$ & e3-tr & e4-SORE-1 & 2 \\
\hline \multirow[t]{5}{*}{ el-as/e3/e4 } & el-as & e2 & e3-ns & e4-SORE-1 & 1 \\
\hline & el-as & e2 & e3-tr & e4-SORE-1 & 8 \\
\hline & el-as & e2 & e3-tr & e4-kes & 4 \\
\hline & el-as & $e 2$ & e3-fs & e4-SORE-1 & 3 \\
\hline & el-as & e2 & e3-fs & e4-kes & 10 \\
\hline \multirow[t]{4}{*}{ El/e3/e4 } & El & $e 2$ & e3-tr & e4-SORE-1 & 4 \\
\hline & El & $e 2$ & e3-tr & e4-oto & 1 \\
\hline & El & $e 2$ & e3-tr & e4-kes & 1 \\
\hline & El & $e 2$ & e3-tr & e4-kam & 2 \\
\hline el/E3/e4 & $e l-n l$ & e2 & E3 & e4-SORE-1 & 2 \\
\hline \multirow[t]{2}{*}{$e 1 / e 3 / E 4$} & $e l-n l$ & $e 2$ & e3-tr & E4 & 7 \\
\hline & el-fs & e2 & e3-tr & E4 & 1 \\
\hline \multirow[t]{2}{*}{ el-as/e3/E4 } & el-as & $e 2$ & e3-tr & E4 & 3 \\
\hline & el-as & e2 & e3-fs & E4 & 4 \\
\hline
\end{tabular}

1) lower-case letters (el,e3 and $e 4)$ indicate loss-of-function alleles at each locus, collectively.

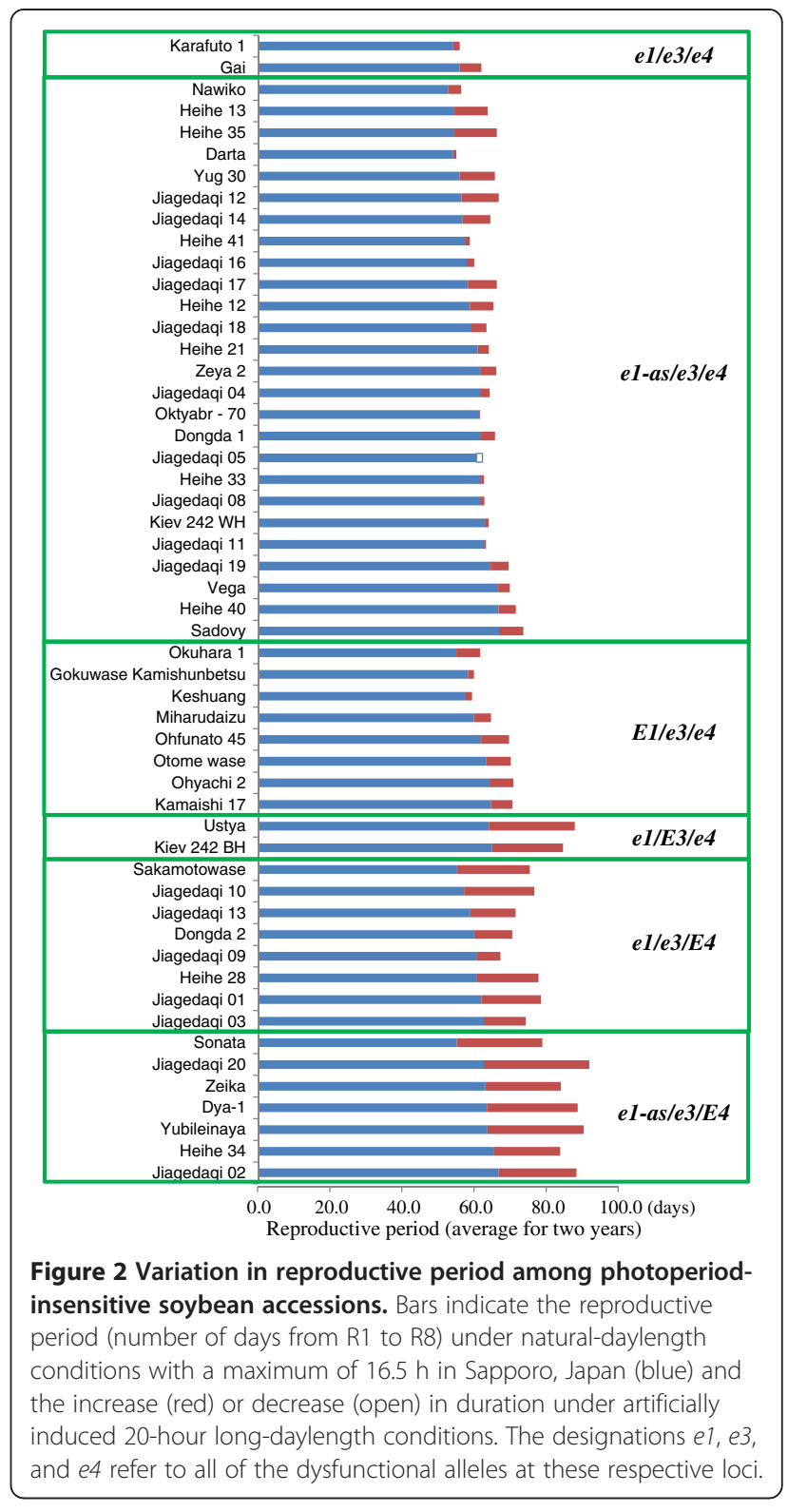

The date of maturation (stage R8 [28]) was delayed more than 5 days during LD in 36 of the 53 accessions tested. This delay was accompanied by slower development of pods or by extension of the flowering period (or both) due to the persistent vegetative activity of apical meristems at the main stem and branches during LD. The difference in reproductive period between ND and LD conditions, evaluated as the number of days from R1 to $\mathrm{R} 8$, ranged from -2 to 29 days and varied with the multi-locus genotypes of the accessions (Figure 2; indicated by blue bars with small red bars [increase under LD] or open bars [decrease under LD]). Among the accessions of the e3/e4 genotype, the delay of maturation during LD was a maximum of 12 days, with an average of 4.2 days. In contrast, the reproductive period was 


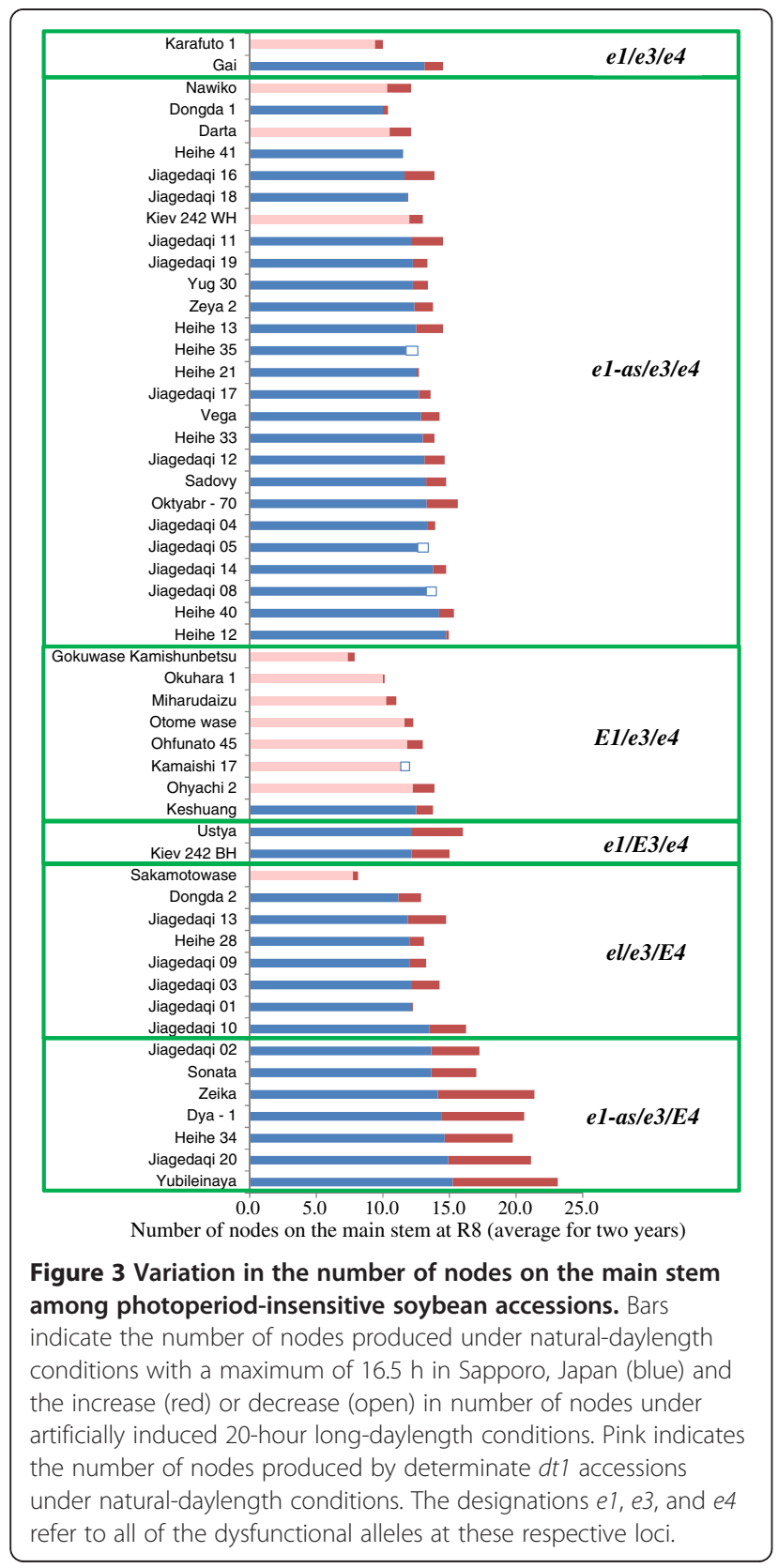

elongated by 12 days or more during LD compared with ND in 14 of the 17 accessions having either the E3 or E4 allele, with an average of 19.1 days. The difference in reproductive period between ND and LD was, on average across accessions and years, 21.8, 14.3, and 23.7 days in the genotypic groups of $e 1(e 1-n l) / E 3 / e 4, e 1(e 1-n l / e 1-f s) /$ $e 3 / E 4$, and $e 1-a s / e 3 / E 4$, respectively (Figure 4 ). This result suggests that E3 and E4 were involved in photoperiodic control not only of time to flowering but also of time to pod maturation.

Daylength also influenced stem termination after flowering in soybean (Figure 3). Stem termination in soybean is known to be controlled by at least two genes, $D t 1$ and
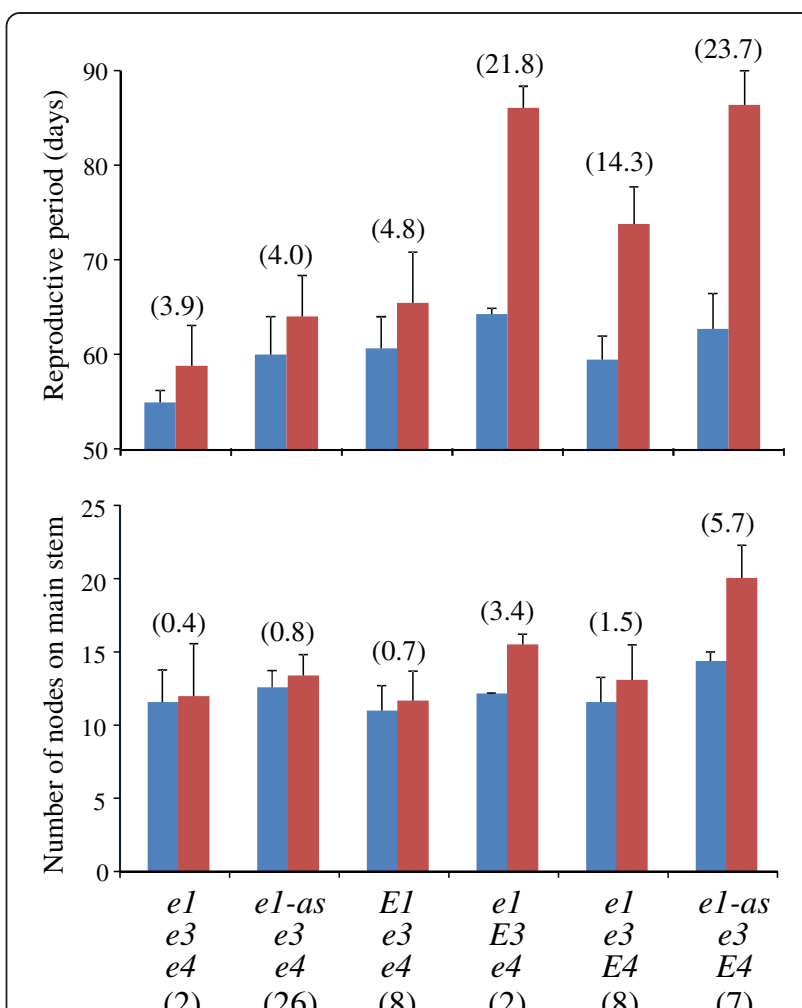

Figure 4 Average and 1 standard deviation (vertical bars) of reproductive period and number of nodes on main stem under ND (blue bars) and LD (red bars) in photoperiod-insensitive soybean accessions of different maturity genotypes. The difference between ND and LD is given in parentheses. The genotypic class E1/e3/e4 mostly consisted of determinate $d t 1$ accessions. The designations e $1, e 3$, and e4 refer to all of the dysfunctional alleles at these respective loci. Numerals within parentheses under genotypes indicate the number of accessions classified into each multi-locus genotype.

$D t 2$, of which the former has a much greater effect [32]. The Dt1 gene is an ortholog of Arabidopsis TERMINAL FLOWER1 (TFL1), GmTFL1b [33,34]. Four dysfunctional alleles (dt1-ab, dt1-bb, dt1-tb, and $d t 1-t a)$ have been identified at this locus; all of these mutant alleles produce proteins containing single amino acid substitutions [34]. Genotyping using allele-specific markers revealed that, of the 53 accessions, 41 had the $D t 1$ allele, and 12 had the $d t 1-b b$ or $d t 1-t b$ allele (Additional file 1 ).

Determinate cultivars typically terminate stem growth shortly after flowering, whereas indeterminate ones continue stem growth until vegetative growth of the shoot apical meristem (SAM) ends [33]. As expected, our 12 determinate accessions that had the $d t 1-b b$ or $d t 1-t b$ allele produced almost the same number of nodes on the main stem during ND and LD (Figure 3; indicated by pink bars with small red or open bar). In contrast, the 41 indeterminate $D t 1$ accessions produced more nodes during LD than during ND, and the differences in node number on 
the main stem between ND and LD varied with the multi-locus genotype (Figure 3; indicated by blue bars with small red or open bar). The difference was less than 2.4 (average, 0.8) nodes among indeterminate accessions with the e3/e4 genotype and was independent of the $E 1$ genotype (Figure 3 ); that is, stem growth terminated shortly after flowering under both ND and LD conditions. In contrast, accessions with the E4 allele tended to produce more nodes during LD than during $\mathrm{ND}$, and this response appeared to be dependent on the genotype (e1 or e1-as) at the E1 locus (Figures 3 and 4). Accessions having the $e 1(e 1-n l)$ allele $(e 1 / e 3 / E 4)$ terminated stem growth similarly between ND and LD; the difference in node number between ND and LD was, on average, 1.5 nodes across accessions and years (Figure 4). In contrast, accessions having the $e 1$-as allele (e1-as/e3/E4) produced at least 3.4 (average, 5.7) more nodes during LD than during ND. Therefore, post-flowering photoperiod growth in soybean, assessed as maturation and stem termination, varied among the accessions tested and with the maturity genotype.

\section{Control of post-flowering stem termination and pod development by E3 and E4}

Han et al. [16] revealed that the post-flowering growth of a photoperiod-sensitive cultivar is influenced by the photoreaction that can be induced by exposure to $\mathrm{R}$ light and reversed by subsequent FR light exposure during the dark period. This pattern implies that phytochromes are involved in the photoperiod responses for postflowering development in soybean. To confirm the different responses of post-flowering growth to photoperiod that we observed among the maturity genotypes in the field experiment, we analyzed pod development, stem termination, and the expression pattern of Dt1 in stem tips under LD for Harosoy (E3/E4/Dt1) and its NILs for e3, e4, and $d t 1$. We induced flowering in these lines as described in the Methods section; SD treatment until 12 days after emergence (DAE) is sufficient to initiate and maintain flowering of Harosoy under non-inductive LD conditions [14]. The soybean maturity genes, $E 3$ and $E 4$, have been characterized in detail for the responses to long days with different $\mathrm{R}: F R$ ratios $[5,9]$. We thus used two LD conditions with different light sources, R-light-enriched and FR-light-enriched, to discriminate the functions of $E 3$ and $E 4$. Harosoy and its NILs for $e 3, e 4$, and $d t 1(d t 1-b b)$ flowered at almost the same time (11 to 13 days after the conversion to LD conditions; Figure 5C, D) under two lighting conditions. Stem growth terminated at the 6th to 8th node in the Harosoy NIL for $d t 1(\mathrm{H}-d t 1)$; indeterminate lines produced more nodes than did $\mathrm{H}-d t 1$, and the number of nodes produced until the end of experiment (30 days after flowering) varied with the maturity genotype (Figure 5A). Compared with $\mathrm{H}-d t 1$, Harosoy and $\mathrm{H}-e 4$ produced, on average, 7.5 and 6.4 more nodes under the R-light-enriched condition and 5.9 to 6.5 nodes more under the FR-light-enriched condition, respectively. $\mathrm{H}-e 3 / e 4$ and $\mathrm{H}-e 3$ terminated stem growth earlier than did Harosoy and $\mathrm{H}-\mathrm{e} 4$ under both conditions. In addition, stem growth terminated earlier in $\mathrm{H}-e 3 / e 4$ than in $\mathrm{H}-e 3$ under the FR-light-enriched condition, and $\mathrm{H}-e 3 / e 4$ produced almost the same number of nodes as did $\mathrm{H}-d t 1$.

Pod development showed a pattern slightly different from the results observed for stem growth (Figure 5B). Under the R-light-enriched condition, Harosoy, H-e4, and $\mathrm{H}-d t 1$ did not produce any pods longer than $3.0 \mathrm{~cm}$ until 30 days after flowering, although they flowered at almost the same time as did $\mathrm{H}-e 3$ and $\mathrm{H}-e 3 / e 4$. In contrast, $\mathrm{H}-e 3$ and $\mathrm{H}-e 3 / e 4$ produced, on average, 5.3 and $3.93-\mathrm{cm}$ or longer pods under the R-light-enriched condition. In contrast, under the FR-light-enriched condition, $\mathrm{H}-e 3$ and $\mathrm{H}-e 3 / e 4$ produced 4.1 and 4.5 pods, respectively, whereas Harosoy yielded only a few pods (0.5), and H-e4 made no pods; $\mathrm{H}-d t 1$ produced an average of 2.6 pods (Figure 5B). The pod development of $\mathrm{H}-d t 1$ in the FRlight-enriched condition may indicate that an R-lightenriched condition has a larger effect in controlling the development of pods after flowering than does the FRlight-enriched condition.

Dt1 expression in determinate soybean plants rapidly decreases concomitant with floral induction, whereas this expression is maintained for a while after flowering starts in indeterminate accessions [33]. Here we found that the expression of Dt1 in the stem tips of 12-day-old plants grown in SD was very low under both R- and FR-enriched conditions, regardless of the genotype at the E3 and E4 loci (Figure 5C and 5D; see data regarding 0 day). In addition, the expression level in $\mathrm{H}-d t 1$ remained very low at later growth stages. In contrast, the indeterminate lines (except $\mathrm{H}-e 3 / e 4$ ) exhibited rapid increases in Dt1 expression at 7 days after conversion to LD (19-day-old plants; 7 days in Figure 5C and 5D). Thereafter, Harosoy and $\mathrm{H}-\mathrm{e} 4$ maintained $D t 1$ expression at relatively high levels until 21 days after conversion to LD under the R-lightenriched condition (Figure $5 \mathrm{C}$ ), but Dt1 expression decreased rapidly at 14 days and afterward under the FR-light-enriched condition (Figure 5D). H-e3 had a similar but slightly lower level of expression than did Harosoy and H-e4 under the FR-light-enriched condition (Figure 5D), but the abundance of Dt1 transcripts decreased rapidly at 14 days after conversion to LD in the R-light-enriched condition (Figure 5C). In contrast to that in Harosoy, H-e3, and H-e4, Dt1 expression was low (similar to that in $\mathrm{H}-d t 1$ ) in $\mathrm{H}-e 3 / e 4$ throughout all growth stages. These results therefore suggest that $D t 1$ expression is under the control of two phyA genes, $E 3$ and $E 4$, for control of post-flowering growth, although a direct causative relationship between $D t 1$ 


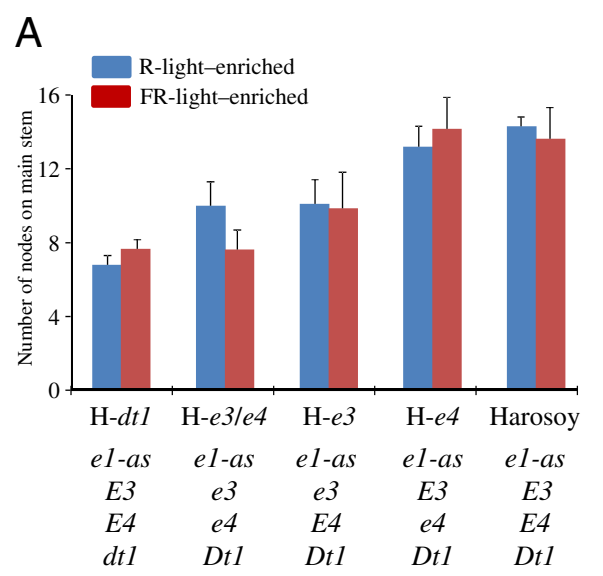

C

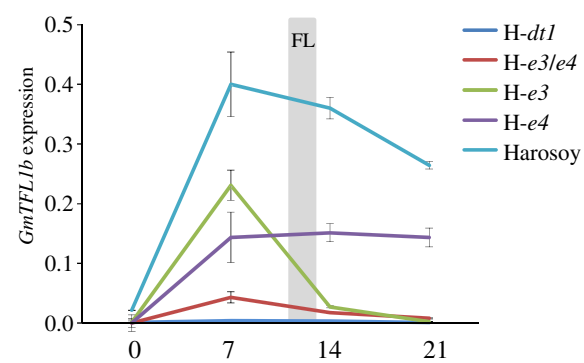

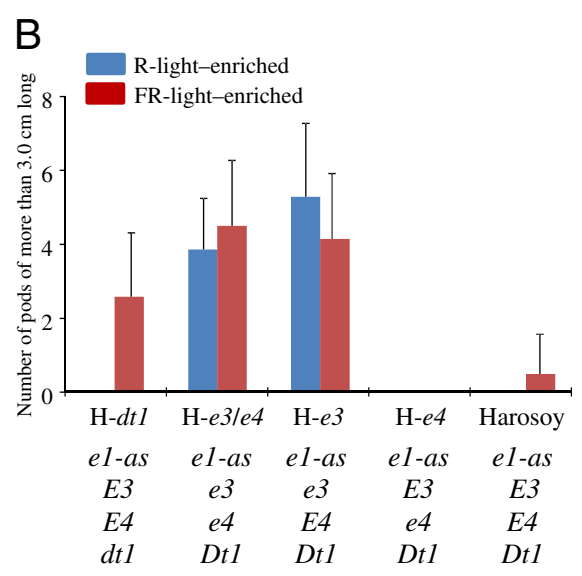

D

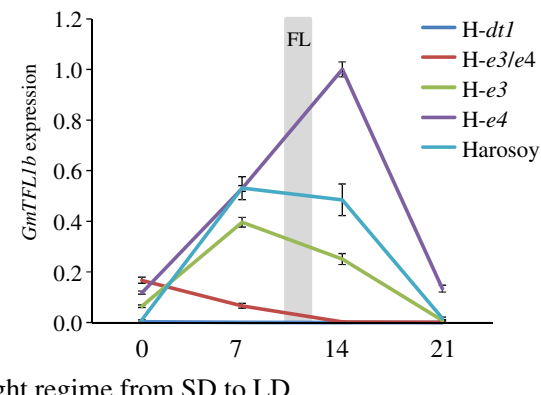

Figure 5 Different post-flowering photoperiod responses among Harosoy and its NILs for a determinate growth habit allele (dt1) and the maturity alleles $e 3$ and $e 4$ in two independent experiments using different light sources, R-light-enriched [blue] and FR-light-enriched [red] conditions. In the R-light-enriched condition, a combination of fluorescent and incandescent lamps with an R:FR ratio of 1.2 was used as the light source for 16 hours after dawn followed by lighting with the same light source for an additional 4 hours. The FR-light-enriched condition involved the use of both fluorescent and incandescent lamps with an R:FR ratio of 1.2 for 16 hours after dawn followed by lighting with incandescent lamps only for an additional 4 hours. A) Number of nodes (average \pm 1 standard deviation) on the main stem at 30 days after flowering. B) Number of pods (average \pm 1 standard deviation) more than $3.0 \mathrm{~cm}$ long at 30 days after flowering. Transcripts levels of Dt1 (GmTFL1b) at stem tips in different growth stages under (C) R-light-enriched and (D) FR-light-enriched conditions. Relative transcript levels (mean and standard error; $n=3$ ) were analyzed by quantitative RT-PCR and normalized to $\beta$-tubulin (TUB). FL indicates time of flowering.

expression and the two phyA genes should be addressed in a further study. This notion is in accordance with PHYA-regulated signal transduction of Dt1 expression, which was expected given the presence in the Dt1 promoter region of a sequence identical to the SORLIP1 (sequence overrepresented in light-induced promoter 1 cis-element [33]). The cis-element sequence found in $D t 1$ is the most common cis-element in SORLIPs in Arabidopsis genes that are induced or repressed by FR light [35].

\section{Discussion}

Three major genetic groups confer photoperiod insensitivity in soybean

In the current study, we assessed the genotypes of 53 photoperiod-insensitive soybean cultivars at four maturity genes and a determinate growth-habit gene by using allele-specific DNA markers that distinguished each of the recessive alleles from the others. These accessions have been introduced from various high-latitude geographic regions. Our first goal was to determine whether there was any association between the genotypes at four major maturity loci (E1 through E4) and photoperiod sensitivity. Our second goal was to reveal any novel genetic factors that controlled photoperiod insensitivity in these diverse genetic resources. The photoperiod-insensitive cultivars and breeding lines we tested were classified into three genotypic groups according to the functions of the alleles at the respective loci: the e3/e4 group; the group containing $e 1(e 1-n l$ or $e 1-f s)$ and either E3 or E4; and the e1-asl e3/E4 group.

All of the 53 photoperiod-insensitive accessions tested had the dysfunctional allele at the $E 2$ locus. The $E 2$ gene is a soybean ortholog of Arabidopsis GIGANTEA (GI) [29]. In Arabidopsis thaliana, GI regulates FT expression through multiple mechanisms: 1) GI binds to FLAVINBINDING, KELCH REPEAT, F BOX protein 1, leading to degradation of a key $\mathrm{CO}$ repressor (CYCLING DOF 
FACTOR 1), upregulation of the expression of CONSTANS (CO), and subsequent activation of $F T$ expression [36,37]; 2) GI regulates the expression levels of miRNA172, whose targets encode repressors of $F T$, such as TARGET OF EAT 1 and SCHLAFMUTZE [38,39]; and 3) GI modulates the stability or promoter accessibility of various $F T$ repressors [40], including SHORT VEGETATIVE PHASE [41], TEMPRANILLO (TEM) 1, and TEM2 [42] Accordingly, the dysfunctional allele at the E2 locus likely is required for photoperiod insensitivity in these soybean accessions.

One of the three genotypic groups consisted of accessions that possessed the double-recessive genotype (e3/e4) at the $E 3$ and $E 4$ loci. These accessions accounted for approximately $70 \%$ (36 of 53 ) of the photoperiod-insensitive accessions tested, suggesting that dysfunction of PHYA is the most common mechanism underlying photoperiod insensitivity in soybean.

Another genotypic group comprised the accessions having the dysfunctional $e 1$ alleles (e1-nl or $e 1-f s)$ in combination with either a dominant E3 or E4 allele. This group contained Sakamotowase, which has the $e 1-f s$ allele that yields a nonfunctional E1 protein, as has been revealed through transient expression assays [13]. In addition, the major QTL for photoperiod insensitivity in this line is tightly linked to the E1 locus [15]. These observations suggest that the photoperiod insensitivity of Sakamotowase can be ascribed to the complete lack of E1 function. Another dysfunctional allele, e1-nl, which lacks the entire $E 1$ gene [13], occurred fairly frequently (11 of 53 lines) among the photoperiod-insensitive accessions we tested. The dysfunctional alleles $e 1-f s$ and $e 1-n l$ may have similar roles in abolishing or weakening the photoperiod responses regulated by the E3 or E4 allele. Additional research is needed to determine whether these dysfunctional alleles at the $E 1$ locus can singly lead to photoperiod insensitivity in the presence of the $E 3$ or $E 4$ allele.

The remaining genotypic group consisted of the accessions with the allelic combination of e1-as/e3/E4. The Harosoy NIL for $e 3$ also has the same allelic combination. However, this NIL is sensitive to the LD conditions generated by using an FR-enriched light source (like that we generated by using incandescent lamps in the current study), although it did not respond to the R-enriched LD condition [4-6,9]. Therefore, the allelic combination of e1-as/e3/ E4 is not sufficient to confer photoperiod insensitivity. It is conceivable that a novel gene-different from the dysfunctional alleles at the E1,E3, and E4 loci-may be involved in the control of the photoperiod insensitivity of accessions of this genotype under the FR-enriched LD condition. GmphyA1, a homoeolog of E4, is a possible candidate for this photoperiod-insensitivity controller: GmphyA1 has been suggested to function in a redundant manner to the $E 4$ allele in the de-etiolation responses of hypocotyls and in flowering under the FR-enriched LD condition [7,9]. The dysfunctional allele at the E4 locus has adverse effects on plant morphogenesis, such as an impaired de-etiolation response under FR light [7] and the production of longer internodes under $\mathrm{LD}$ conditions relative to the $E 4$ allele [43], making the plant susceptible to lodging. Accordingly, the use of the dominant E4 allele may contribute to the lodging tolerance of photoperiodinsensitive cultivars if another factor conditions the insensitivity. An appropriate combination of alleles should therefore be selected from among those representing diverse genetic mechanisms to adapt soybean cultivars to the conditions of the target environment.

Multiple dysfunctional alleles have been detected for all of the $E 1, E 3$, and $E 4$ loci $[13,31]$, including two novel alleles at the $E 3$ locus [this study]. Our previous studies indicated that five loss-of-function alleles at the E4 locus originated recently and independently in different soybean landraces from East Asia [31,44]. These results suggest that photoperiod insensitivity in soybean has arisen redundantly through multiple combinations of independently generated alleles at the E1,E3, and E4 loci.

\section{Two phyA genes, E3 and E4, control post-flowering responses in soybean}

Another finding of the current study is that vegetative and reproductive growth characteristics after flowering, such as maturation and stem termination, were influenced by photoperiod responses regulated by the E3 and E4 loci. Our findings are therefore in good agreement with previous observations regarding an association between photoperiod sensing and post-flowering responses [16-18] and involvement of phytochrome(s) in post-flowering responses [16]. We found that soybean accessions with different photoperiod-insensitive multi-locus genotypes responded differently to the photoperiod after flowering. Despite their photoperiod insensitivity in regard to time to flowering, accessions that had either the $E 3$ or $E 4$ allele matured at least 10 days later under LD than ND conditions (Figures 2 and 4). Furthermore, vegetative activity of the SAM during LD persisted through late growth stages in the indeterminate accessions with allelic combinations of $e 1 / E 3$ or $e 1$-as/E4 to produce more nodes than did those grown under ND condition (Figures 3 and 4). These behaviors are in sharp contrast to those of the accessions of the e3/e4 group, which, independent of the type of allele or genotype at the $E 1$ locus, matured without notable delays during LD relative to ND and terminated stem growth similarly under these two conditions (Figure 4).

The different photoperiod responses of post-flowering vegetative and reproductive growth that we observed among the various maturity genotypes were further confirmed through analyses with Harosoy and its NILs for $e 3$, $e 4$, and $d t 1$. Plants among the NILs in which we induced 
flowering by SD treatment for 12 DAE exhibited different stem and pod growth under subsequent LD conditions. In particular, the NILs with the E3 allele retained the vegetative activity of the SAM of flower-induced plants and inhibited the pod development after flowering during LD conditions, whereas those with a recessive $e 3$ allele terminated stem growth shortly after flowering to produce fewer nodes and developed pods under both of the light regimens (Figures 5A and 5B). We also detected a slight effect of $e 4$ only in the genetic background of $e 3$ under the light regimen in which only FR-enriched lights were used to provide four hours of additional daytime subsequent to the 16-hour daytime period (Figure 5A). Our present results strongly suggest that post-flowering photoperiod responses in soybean are controlled by the E3 and E4 genes.

\section{A proposed gene regulatory network for pre- and post- flowering photoperiod responses in soybean}

We have summarized our current results as a gene network involving three maturity genes $(E 1, E 3$, and $E 4)$, a determinate habit gene (Dt1), and two GmFTs (GmFT2a and $G m F T 5 a$ ), that regulates the pre-flowering and postflowering photoperiod responses of soybean under LD (Figure 6). In photoperiod-sensitive plants having the E1 or e1-as allele and a dominant allele at either the E3 or E4 locus (or both) (Figure 6A), we propose that flowering is not induced under FR-enriched LD [3-5,9] because the $E 1$ and $e 1$-as alleles inhibit the gene expression of GmFT2 $a$ and GmFT5 $a$ [13]. When those plants are exposed to a short period of SD, flowering is induced and persists even after the transfer to non-inductive LD, but subsequent seed maturation is delayed through the influence of an as yet unknown factor (Y), and vegetative activity at SAM is retained to produce more nodes due to $D t 1$ expression. Both factor $\mathrm{Y}$ and $D t 1$ are under the control of PHYA encoded at the E3 or E4 locus (Figure 6A).

We propose that flowering in photoperiod-insensitive plants of the e3/e4 group, which has dysfunctional alleles at the $E 3$ and $E 4$ loci, is induced through the upregulation of GmFTs in the absence of $E 1$ expression [13]. Flowering in e3/e4 plants is followed by normal seed maturation and stem termination, possibly due to downregulation of $\mathrm{Y}$ and $D t 1$, respectively (Figure 6B). The delayed maturity in some of the $e 3 / e 4$ accessions in LD compared with ND (Figure 2 and 4) may be ascribed to the presence of a copy of the phyA gene at a different locus (GmphyA1) [7] or other photoreceptors.

In contrast, in photoperiod-insensitive plants that have a functional phyA gene at either the E3 or E4 locus, we suggest that flowering is induced under LD either by loss-of-function alleles at the E1 locus in the e1/e3/E4$e 1 / E 3 / e 4$ group (Figure $6 \mathrm{C}$ ) or by an unknown genetic factor $(\mathrm{X})$ in the e1-as/e3/E4 group (Figure 6D). In contrast to the e3/e4 group, seed maturation in these two groups is delayed similarly under LD due to regulation by a functional phyA gene (E3 or E4). However, stem growth under LD differs between these groups: stem growth terminates earlier in the $e 1 / e 3 / E 4-e 1 / E 3 / e 4$ group than in the e1-as/e3/E4 group (Figures 3 and 4). In e1-as/ e3/E4 plants, PHYA-mediated Dt1 expression under LD may preserve vegetative activity at SAM to produce more nodes, as indicated by the post-flowering responses of Harosoy and its NILs for $e 3$ or $e 4$ in LD (Figure 5).

These models prompt two questions regarding postflowering growth in soybean. First, how does the E1 gene influence stem growth after flowering? Apart from its effect on flowering and maturity, the role of the E1 gene in morphogenesis has not been determined, although it indeed influences various morphologic traits, yield, and other traits such as tolerance to chilling temperature $[45,46]$. The $E 1$ and $e 1$-as alleles inhibit the expression of GmFT2 $a$ and GmFT5a, which are under the control of phyA genes [13], whereas the loss-of-function alleles $e 1-f s$ and $e 1-n l$ do not. In Arabidopsis, FT protein binds to FLOWERING LOCUS D (FD) and promotes the expression of APETALA1 and $L E A F Y$, flower meristem genes [47-49]; reviewed in [50], which, in turn, suppresses TFL1 transcription and terminates stem growth [51,52]. Therefore, the different effects of the alleles at the E1 locus on post-flowering stem growth might be ascribed not to a direct effect but rather to an indirect effect through suppression of GmFT expression.

Another question is whether flowering itself is a direct trigger for seed development in soybean. The findings we obtained in the current study indicate that PHYAmediated photoperiod responses may regulate seed maturation directly or indirectly via an as yet unknown factor: only when two phyA genes were dysfunctional did seed maturation after flowering under LD proceed without any marked delay (Figure 6). Additional research therefore should explore key factors (such as $\mathrm{Y}$ in our model) in promoting seed development. Comparing expression profiles during early seed development between SD-grown plants and those whose reproductive growth has reverted to a vegetative pattern may advance our understanding of the molecular bases of seed development in soybean.

\section{Conclusions}

The present study revealed diverse genetic mechanisms underlying photoperiod insensitivity in soybean. At least three multi-locus genotypes comprising various allelic combinations at the E1, E3, and E4 loci can confer preflowering photoperiod insensitivity. These genotypes responded differently to photoperiod during post-flowering reproductive development, indicating involvement of the phyA genes E3 and E4. Our results further indicate that 


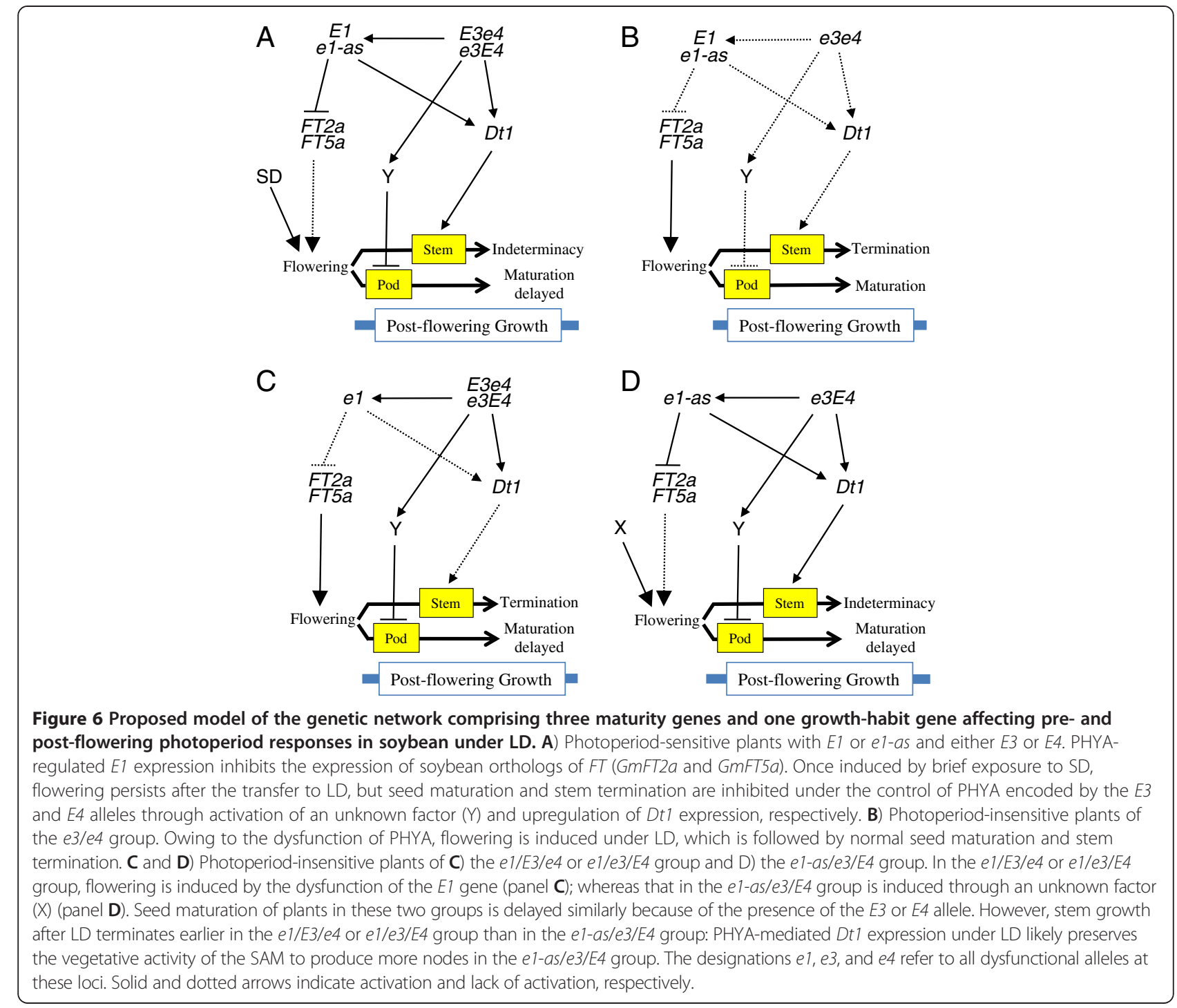

flowering itself may not necessarily be a direct trigger for seed development. E1,E3, and E4 control the photoperiod responses for pre- and post-flowering development, which directly influences final seed yield in soybean.

\section{Methods}

\section{Plant materials}

We used 53 photoperiod-insensitive soybean (Glycine max) accessions in the current study (Additional file 1). These included 9 accessions from northern Japan (6 from Hokkaido and 3 from the Tohoku region), 29 from northeast China, 8 from far-Eastern Russia, 4 from Ukraine, and 3 from Poland. Harosoy (PI548573) and its NILs for e3 (PI547716; $\mathrm{H}-e 3$ ), e4 (PI591435; H-e4), both e3 and e4 (PI546043; $\mathrm{H}-e 3 / e 4$ ), and $d t 1$ (PI547687; $\mathrm{H}-d t 1$ ) were used as controls to delineate pre- and post-flowering photoperiod insensitivity.

\section{Evaluation of photoperiod sensitivity}

Photoperiod sensitivity was evaluated based on differences in the date when the first flower opened (stage R1 [27]) between plants grown under artificially induced LD conditions and those under ND conditions. The experiment was performed in an experimental field with outdoor lighting at Hokkaido University, Sapporo, Japan $\left(43^{\circ} 06^{\prime} \mathrm{N}\right.$, $141^{\circ} 35^{\prime} \mathrm{E}$ ) during 2011 and 2012. ND conditions in Sapporo, including civil twilight, reached a maximum of $16.5 \mathrm{~h}$. Seeds were sown in paper pots (No. 2, Nippon Tensai Tougyo, Obihiro, Japan) on June 1, 2011, and May 29, 2012, and 10-day-old seedlings were transplanted into both LD and ND fields. LD conditions were generated by using 500-W incandescent lamps placed $2 \mathrm{~m}$ above the soil surface at intervals of $4 \mathrm{~m}$. Lights were on from 0300 to 0600 and from 1830 to 2300 until the end of treatment (August 2). Under illumination by incandescent lamps, the R:FR (660:730) quantum ratio was 0.72, and the 
average photosynthetic photon flux at the canopy surfaces was $1 \mu \mathrm{mol}$ photon $\mathrm{sec}^{-1} \mathrm{~m}^{-2}$, as measured at night by using a quantum sensor (model LI-1800C, Li-Cor, Lincoln, NE). The plants were checked every other day to determine stages R1 and R8 [27]. The nodes on the main stem were counted at R8.

\section{Genotyping by using allele-specific DNA markers}

We genotyped four maturity genes (E1 through E4) and a growth habit gene (Dt1) by using allele-specific DNA markers for $E 1$ [13], E2 [29], E3 [30], E4 [7,31], and dt1 $(d t 1-b b)$ [33]. We used available sequence information [34] to develop DNA markers for $d t 1-a b$ and $d t 1-t b$; we did not develop a DNA marker for dt1-ta because of its rarity [34]. Primer sequences, DNA marker type, restriction enzyme used, and resultant fragment sizes for each marker are shown in Additional file 2. Total genomic DNA was extracted from trifoliate leaves as described previously [53]. Each PCR reaction contained $30 \mathrm{ng}$ of total genomic DNA as template and ExTaq polymerase (TaKaRa, Otsu, Japan); amplification conditions were 30 cycles at $94^{\circ} \mathrm{C}$ for $30 \mathrm{sec}, 56$ to $60^{\circ} \mathrm{C}$ (depending on the primers used) for $30 \mathrm{sec}$, and $72^{\circ} \mathrm{C}$ for 30 to $90 \mathrm{sec}$. PCR products or those digested with appropriate restriction enzymes as needed were separated by electrophoresis in $1 \%$ to $3 \%(\mathrm{w} / \mathrm{v})$ agarose gels, stained with ethidium bromide, and visualized under UV light.

\section{Sequence analyses}

Accessions having at least one dominant allele of E1, E3, or $E 4$ were sequenced. A 525 -bp region for $E 1$, four regions of 571 to $2350 \mathrm{bp}$ covering each of four exons for E3, and two overlapping fragments of 2384 and $3506 \mathrm{bp}$ that covered the entire GmphyA2 coding sequence for E4 were amplified from genomic DNA by using KOD FX (Toyobo Life Science, Osaka, Japan). Amplified fragments were purified by using the ExoSAP-IT enzyme kit (GE Life Sciences Japan, Tokyo, Japan). The purified PCR products were used as templates for forward and reverse sequencing reactions generated by using a BigDye Terminator v3.1 Cycle Sequencing kit and ABI PRISM 3100 Avant Genetic Analyzer (Applied Biosystems Japan, Tokyo, Japan) according to the manufacturer's instructions.

\section{Development of DNA markers for novel E3 loss-of- function alleles}

We used sequences flanking mutation sites to develop allele-specific CAPS and dCAPS DNA markers. The targeted region for each mutation was amplified from the DNA preparations by using ExTaq polymerase with primers specific to each mutation. The PCR products were digested with appropriate restriction enzymes (Additional file 2), separated by electrophoresis in $1 \%$ or $2.5 \%$ agarose gels, stained with ethidium bromide, and visualized under UV light.

\section{Expression analysis for the determinate growth habit gene $D t 1$}

Time-course-dependent expression of the Dt1 (GmTFL1b) gene, an Arabidopsis TERMINAL FLOWER1 (TFL1) ortholog, at the stem tip was analyzed for Harosoy and its NILs for $d t 1, e 3$, and e4. Plants were grown in a growth chamber with a constant air temperature of $25^{\circ} \mathrm{C}$, an average photon flux of $300 \mu \mathrm{mol}$ photons $\mathrm{m}^{-2} \mathrm{~s}^{-1}$, and an R:FR ratio of 1.2 provided by a combination of fluorescent and incandescent lamps. The daylength was set at 12 hours until 12 DAE and at 20 hours thereafter. Two independent experiments were done by using different light sources. Specifically, in experiment 1 , a combination of fluorescent and incandescent lamps with an R:FR ratio of 1.2 was used as the light source for 16 hours after dawn followed by lighting with the same light source for an additional 4 hours; experiment 2 involved the use of both fluorescent and incandescent lamps with an R:FR ratio of 1.2 for 16 hours after dawn followed by lighting with incandescent lamps only for an additional 4 hours. In both experiments, stem tips were collected in bulk from four individual plants at 4 zeitgeber time every 7 days beginning at $12 \mathrm{DAE}$. The samples were immediately frozen in liquid $\mathrm{N}_{2}$ and stored at $-80^{\circ} \mathrm{C}$.

Transcript levels of $D t 1$ were determined by quantitative real-time PCR (qRT-PCR). The qRT-PCR mixture was prepared by mixing $1 \mu \mathrm{L}$ of the $\mathrm{CDNA}$ synthesis reaction, $5 \mu \mathrm{L} 1.2 \mu \mathrm{M}$ primer premix, $10 \mu \mathrm{L}$ SYBR Premix ExTaq Perfect Real Time (TaKaRa, Otsu, Japan), and water to yield a final volume of $20 \mu \mathrm{L}$. The analysis was done by using the CFX96 Real-Time System (BIO-RAD Laboratories Japan, Tokyo, Japan). The primers used were 5 ' -AGGCAC AACAGATGCCACAT-3' and 5'-GGCAAAACCAGCA GCTACTT-3' for GmTFL1b (Dt1) and 5'-GAGAAGAG TATCCGGATAGG-3' and 5'-GAGCTTGAGTGTTCGG AAAC-3' for $\beta$-tubulin. The PCR cycling conditions were $95^{\circ} \mathrm{C}$ for $3 \mathrm{~min}$ followed by 40 cycles of $95^{\circ} \mathrm{C}$ for $10 \mathrm{sec}$, $58^{\circ} \mathrm{C}$ for $20 \mathrm{sec}, 72^{\circ} \mathrm{C}$ for $20 \mathrm{sec}$, and $78^{\circ} \mathrm{C}$ for $2 \mathrm{sec}$. Fluorescence was quantified before and after incubation at $78^{\circ} \mathrm{C}$ to monitor the formation of primer dimers. A reaction mixture without reverse transcriptase was included as a control to confirm that no amplification resulted from genomic DNA contaminants in the RNA sample. In all PCR experiments, amplification of a single DNA species was confirmed by both melting curve analysis of qRTPCR and gel electrophoresis of PCR products. The mRNA level of GmTFL1b was normalized to that of $\beta$-tubulin.

\section{Accession numbers}

Sequence data from this article can be found in the GenBank/EMBL/DDBJ data libraries under the accession numbers AB766210 (exon 1 of the $e 3-f_{s}$ GmphyA3 allele) and AB766211 (exon 3 of the e3-ns GmphyA3 allele). 


\section{Additional files}

Additional file 1: Genotypes at four maturity loci and a determinate growth habit locus in 53 photoperiod-insensitive soybean accessions of different origins, as estimated by using allele-specific DNA markers.

Additional file 2: Allele-specific DNA markers that distinguish recessive alleles from dominant functional ones at the maturity loci $E 1, E 2, E 3$, and $E 4$ and the determinate growth habit locus Dt1 in soybean.

\section{Abbreviations}

QTL: Quantitative trait locus; NIL: Near-isogenic line; PCR: Polymerase chain reaction; qRT-PCR: Quantitative real-time PCR; CAPS: Cleaved amplified polymorphic sequence; dCAPS: Derived CAPS; PHYA: Phytochrome A; FT: FLOWERING LOCUS T; GI: GIGANTEA; CO: CONSTANS; TFL1: TERMINAL FLOWER1; SAM: Shoot apical meristem; DAS: Days after sowing.

\section{Competing interests}

The authors declare that they have no competing interests.

\section{Authors' contributions}

$M X$ and $Z X u$ contributed equally to this research. $M X, Z X u, B L$, and JA conducted the experiment. MX, BL, and JA classified the photoperiod sensitivity of accessions. MX and Z Xu conducted sequencing analyses. MX Z Xia, FK, YT, and SW developed allele-specific DNA markers and analyzed the variation of genotypes in soybean accessions. MX and TY conducted the expression analyses. MX and JA drafted the manuscript with edits from $\mathrm{FK}, \mathrm{BL}, \mathrm{SW}, \mathrm{AK}$, and $\mathrm{KH}$. All authors read and approved the final manuscript.

\section{Acknowledgments}

The authors are grateful to Drs. AY Ala (All Russian Research Institute of Soybean, Russia), G Konieczny (Agricultural University of Poznan, Poland), VI Sichkar (Institute of Plant Breeding and Genetics, Ukraine), VG Mikhailov and O Hrabovsky (Institute of Agriculture of Ukrainian Academy of Agrarian Sciences, Ukraine), and ER Cober (Agriculture and Agri-Food Canada, Canada) for providing us seeds of soybean cultivars and experimental lines. This work was supported in part by Grants-in-Aid for Scientific Research from the Ministry of Education, Culture, Sports, Science, and Technology of Japan (23380001) to J Abe; by the National Science Foundation of China (grant nos. 30971813, 31071445) and the Program of "One Hundred Talented People" of the Chinese Academy of Sciences (grant no. KZCX2-YW-BR-11) to B Liu; and by the Natural Science Foundation of China (grant no. 31071445) and the Program of "One Hundred Talented People" of Chinese Academy of Sciences to F Kong.

\section{Author details}

${ }^{1}$ Research Faculty of Agriculture, Hokkaido University, Sapporo 060-8589, Japan. ${ }^{2}$ National Institute of Agrobiological Sciences, Tsukuba, Ibaraki 305-8602, Japan. ${ }^{3}$ Northeast Institute of Geography and Agroecology, Chinese Academy of Sciences, Harbin 150040, China.

Received: 2 March 2013 Accepted: 18 June 2013

Published: 25 June 2013

\section{References}

1. Watanabe S, Harada K, Abe J: Genetic and molecular bases of photoperiod responses of flowering in soybean. Breed Sci 2012, 61:531-543.

2. Buzzell Rl: Inheritance of a soybean flowering response to fluorescentdaylength conditions. Can J Genet Cytol 1971, 13:703-707.

3. Buzzell Rl, Voldeng HD: Inheritance of insensitivity to long day length. Soybean Genet News/ 1980, 7:26-29.

4. Saindon G, Voldeng HD, Beversdorf WD, Buzzell Rl: Genetic control of long daylength response in soybean. Crop Sci 1989, 29:1436-1439.

5. Cober ER, Tanner JW, Voldeng HD: Genetic control of photoperiod response in early-maturing near-isogenic soybean lines. Crop Sci 1996, 36:601-605.

6. Abe J, Xu DH, Miyano A, Komatsu K, Kanazawa A, Shimamoto Y: Photoperiod-insensitive Japanese soybean landraces differ at two maturity loci. Crop Sci 2003, 43:1300-1304.
7. Liu B, Kanazawa A, Matsumura H, Takahashi R, Harada K, Abe J: Genetic redundancy in soybean photoresponses associated with duplication of phytochrome A gene. Genetics 2008, 180:996-1007.

8. Watanabe S, Hideshima R, Xia Z, Tsubokura Y, Sato S, Nakamoto Y, Yamanaka N, Takahashi R, Ishimoto M, Anai T, Tabata S, Harada K: Map-based cloning of the gene associated with the soybean maturity locus E3. Genetics 2009, 182:1251-1262.

9. Cober ER, Tanner JW, Voldeng HD: Soybean photoperiod-sensitivity loci respond differentially to light quality. Crop Sci 1996, 36:606-610.

10. Franklin KA, Allen T, Whitelam GC: Phytochrome $A$ is an irradiance-dependent red light sensor. Plant J 2007, 50:108-117.

11. Franklin KA, Whitelam GC: Phytochrome $A$ function in red light sensing. Plant Signal Behav 2007, 2:383-385.

12. Casal JJ, Sanchez RA, Yanovsky MJ: The function of phytochrome A Plant Cell Environ 1997, 20:813-819.

13. Xia Z, Watanabe $S$, Yamada T, Tsubokura $Y$, Nakashima H, Zhai H, Anai T, Sato S, Yamazaki T, Lü S, Wu H, Tabata S, Harada K: Positional cloning and characterization reveal the molecular basis for soybean maturity locus $E 1$ that regulates photoperiodic flowering. Proc Natl Acad Sci USA 2012, 109:E2155-64.

14. Kong F, Liu B, Xia Z, Sato S, Kim BM, Watanabe S, Yamada T, Tabata S, Kanazawa A, Harada K, Abe J: Two coordinately regulated homologs of FLOWERING LOCUS T are involved in the control of photoperiodic flowering in soybean. Plant Physiol 2010, 154:1220-1231.

15. Liu B, Abe J: QTL Mapping for photoperiod-insensitivity of a Japanese soybean landrace Sakamotowase. J Hered 2010, 101:251-256.

16. Han T, Wu C, Tong Z, Mentreddy RS, Tan K, Gai J: Postflowering photoperiod regulates vegetative growth and reproductive development of soybean. Env Exp Bot 2006, 55:120-129.

17. Kantolic AG, Slafer GA: Development and seed number in indeterminate soybean as affected by timing and duration of exposure to long photoperiods after flowering. Ann Bot 2007, 99:925-933.

18. Jiang $Y, W u C$, Zhang $L, H u P, H o u$ W, Han T: Long-day effects on the terminal inflorescence development of a photoperiod-sensitive soybean [Glycine max (L.) Merr.] variety. Plant Sci 2010, 180:504-10.

19. Bernard RL: Two genes for time of flowering in soybeans. Crop Sci 1971, 11:242-244.

20. McBlain BA, Hesketh JD, Bernard RL: Genetic effect on reproductive phenology in soybean isolines differing in maturity genes. Can J Plant Sci 1987, 67:105-116.

21. Saindon G, Beversdorf WD, Voldeng HD: Adjusting of the soybean phenology using the E4 loci. Crop Sci 1989, 29:1361-1365.

22. Lee SH, Bailey MA, Mian MAR, Shipe ER, Ashley DA, Parrot PW, Hussey RS, Boerma HR: Identification of quantitative trait loci for plant height, lodging, and maturity in a soybean population segregating for growth habit. Theor Appl Genet 1996, 92:516-523.

23. Watanabe S, Tajuddin T, Yamanaka N, Hayashi M, Harada K: Analysis of QTLs for reproductive development and seed quality traits in soybean using recombinant inbred lines. Breed Sci 2004, 54:399-407.

24. Zhang WK, Wang YJ, Luo GZ, Zhang JS, He CY, Wu XL, Gai JY, Chen SY: QTL mapping of ten agronomic traits on the soybean (Glycine max L. Merr.) genetic map and their association with EST markers. Theor Appl Genet 2004, 108:1131-1139.

25. Cheng L, Wang Y, Zhang C, Wu C, Xu J, Zhu H, Leng J, Bai Y, Guan R, Hou W, Zhang L, Han T: Genetic analysis and QTL detection of reproductive period and post-flowering photoperiod responses in soybean. Theor App/ Genet 2011, 123:421-429.

26. Komatsu K, Hwang TY, Takahashi M, Sayama T, Funatsuki H, Oki N, Ishimoto M: Identification of QTL controlling post-flowering period in soybean. Breed Sci 2012, 61:646-652.

27. Cober ER, Molnar SJ, Charette M, Voldeng HD: A new locus for early maturity in soybean. Crop Sci 2010, 50:524-527.

28. Fehr WR, Caviness CE, Burmood DT, Pennington JS: Stage of development description for soybeans, Glycine max (L.) Merrill. Crop Sci 1971, 11:929-931.

29. Watanabe S, Xia Z, Hideshima R, Tsubokura Y, Sato S, Harada K: A map-based cloning strategy employing a residual heterozygous line reveals that the GIGANTEA gene is involved in soybean maturity and flowering. Genetics 2011, 188:395-407

30. Harada K, Watanabe S, Xia Z, Tsubokura Y, Yamanaka N, Anai T: Positional cloning of the responsible genes for maturity Loci $E 1, E 2$ and $E 3$ in 
soybean. In Soybean-Genetics and Novel Techniques for Yield Enhancement. Edited by Krezhova D. InTech; 2011:51-76.

31. Tsubokura Y, Matsumura H, Xu M, Nakashima H, Liu B, Anai T, Kong F, Yuan X, Kanamori H, Katayose Y, Takahash R, Harada K, Abe J: Genetic variation in soybean at the maturity locus $E 4$ is involved in adaptation to long days at high latitudes. Agronomy 2013, 3:117-134.

32. Bernard RL: Two genes affecting stem termination in soybean. Crop Sci $1972,12: 235-239$

33. Liu B, Watanabe S, Uchiyama T, Kong F, Kanazawa A, Xia Z, Nagamatsu A, Arai M, Yamada T, Kitamura K, Masuta C, Harada K, Abe J: The soybean stem growth habit gene Dt1 is an ortholog of Arabidopsis TERMINAL FLOWER 1. Plant Physiol 2010, 153:198-210.

34. Tian Z, Wang X, Lee R, Li Y, Specht JE, Nelson RL, McClean PE, Qiu L, Ma J: Artificial selection for determinate growth habit in soybean. Proc Natl Acad Sci USA 2010, 107:8563-8568.

35. Hudson MF, Quail PH: Identification of promoter motifs involved in the network of phytochrome A-regulated gene expression by combined analysis of genomic sequences and microarray data. Plant Physio/ 2003, 133:1605-1616.

36. Mizoguchi T, Wright L, Fujiwara S, Cremer F, Lee K, Onouchi H, Mouradov A, Fowler S, Kamada H, Putterill J, Coupland G: Distinct roles of GIGANTEA in promoting flowering and regulating circadian rhythms in Arabidopsis. Plant Cell 2005, 17:2255-2270.

37. Sawa M, Nusinow DA, Kay SA, Imaizumi T: FKF1 and GIGANTEA complex formation is required for day-length measurement in Arabidopsis. Science 2007, 318:261-265.

38. Jung JH, Seo YH, Seo PJ, Reyes JL, Yun J, Chua NH, Park CM: The GIGANTEA-regulated microRNA172 mediates photoperiodic flowering independent of CONSTANS in Arabidopsis. Plant Cell 2007, 19:2736-2748.

39. Mathieu J, Yant LJ, Mürdter F, Küttner F, Schmid M: Repression of flowering by the miR172 target SMZ. PLoS Biol 2009, 7:e1000148.

40. Sawa M, Kay SA: GIGANTEA directly activates Flowering Locus $T$ in Arabidopsis thaliana. Proc Natl Acad Sci USA 2011, 108:11698-11703.

41. Lee JH, Yoo SJ, Park SH, Hwang I, Lee JS, Ahn JH: Role of SVP in the control of flowering time by ambient temperature in Arabidopsis. Genes Dev 2007, 21:397-402.

42. Castillejo C, Pelaz S: The balance between CONSTANS and TEMPRANILLO activities determines FT expression to trigger flowering. Curr Biol 2008 18:1338-1343

43. Abe J: Genetic diversity and its use in soybean. In Proceedings of the $14^{\text {th }}$ NIAS International Workshop on Genetic Resources 'Genetic Resources and Comparative Genomes of Legumes (Glycine and Vigna). Edited by Tomooka N, Vaughan DA. Tsukuba: Kobe, Sato Printing Co., Ltd; 2011:91-97.

44. Kanazawa A, Liu B, Kong F, Arase S, Abe J: Adaptive evolution involving gene duplication and insertion of a novel Ty1/copia-like retrotransposon in soybean. $J \mathrm{Mol}$ Evol 2009, 69:164-175.

45. Takahashi R, Abe J: Soybean maturity genes associated with seed coat pigmentation and cracking in response to low temperatures. Crop Sci 1999, 39:1657-1662.

46. Githiri SM, Yang D, Khan NA, Xu D, Komatsuda T, Takahashi R: QTL analysis of low temperature induced browning in soybean seed coats. $J$ Hered 2007, 98:360-366.

47. Abe M, Kobayashi Y, Yamamoto S, Daimon Y, Yamaguchi A, Ikeda Y, Ichinoki H, Notaguchi M, Goto K, Araki T: FD, a bZIP protein mediating signals from the floral pathway integrator FT at the shoot apex. Science 2005, 309:1052-1056.

48. Wigge PA, Kim MC, Jaeger KE, Busch W, Schmid M, Lohmann JU, Weigel D: Integration of spatial and temporal information during floral induction in Arabidopsis. Science 2005, 309:1056-1059.

49. Huang T, Böhlenius H, Eriksson S, Parcy F, Nilsson O: The mRNA of the Arabidopsis gene FT moves from leaf to shoot apex and induces flowering. Science 2005, 309:1694-1696.

50. Sablowski R: Flowering and determinacy in Arabidopsis. J Exp Bot 2007, 58:899-907
51. Liljegren SJ, Gustafson-Brown C, Pinyopich A, Ditta GS, Yanofsky MF: Interactions among APETALA1, LEAFY, and TERMINAL FLOWER1 specify meristem fate. Plant Cell 1999, 11:1007-1018.

52. Ratcliffe OJ, Bradley DJ, Coen ES: Separation of shoot and floral identity in Arabidopsis. Development 1999, 126:1109-1120.

53. Doyle JJ, Doyle JL: Isolation of plant DNA from fresh tissue. Focus 1990 $12: 13-15$.

doi:10.1186/1471-2229-13-91

Cite this article as: Xu et al:: Genetic variation in four maturity genes affects photoperiod insensitivity and PHYA-regulated post-flowering responses of soybean. BMC Plant Biology 2013 13:91.

\section{Submit your next manuscript to BioMed Central and take full advantage of:}

- Convenient online submission

- Thorough peer review

- No space constraints or color figure charges

- Immediate publication on acceptance

- Inclusion in PubMed, CAS, Scopus and Google Scholar

- Research which is freely available for redistribution

Submit your manuscript at www.biomedcentral.com/submit
C) BioMed Central 